\title{
Sirt1-Independent Rescue of Muscle Regeneration by Resveratrol in Type I Diabetes
}

\author{
Jaemin Jeong $^{1,2 *}$, Michael J. Conboy ${ }^{1}$ and Irina M. Conboy ${ }^{1 *}$ \\ ${ }^{1}$ Department of Bioengineering and QB3 Institute, University of California, USA \\ ${ }^{2}$ Division of Radiation Oncology, Korea institute of Radiological and Medical Sciences, South Korea
}

\begin{abstract}
The loss of insulin producing pancreatic $\beta$-cells or peripheral resistance to insulin signaling causes diabetes mellitus, a terminal metabolic disorder that broadly affects organ systems ultimately leading to chronic wounds, blindness, muscle wasting and other disorders of abandoned tissue maintenance. We have recently demonstrated that in a mouse model of type I diabetes, there is a rapid decline in the regenerative capacity of muscle stem (satellite) cells due to elevated levels of myostatin; and that either insulin or inhibition of TGF- $\beta$ receptor signaling rescues muscle repair in the diabetic condition. Resveratrol (RSV) has been shown to improve energy metabolism in cases of type II diabetes, but its effects on stem cells and tissue regeneration are not well understood in a diabetic organism in general or in type I diabetes in particular. In this work, we studied the effect of RSV on the enhancement of muscle regeneration in type I diabetes. Our data demonstrate that systemic delivery of RSV to severely diabetic mice with ablated pancreatic $\beta$-cells rescues muscle regeneration by boosting the myogenic capacity of muscle stem cells. Therefore, our results suggest that in our experimental system, RSV did not reduce the blood glucose levels (BGL) in diabetic animals and did not act through its key effector Sirt1 in the rescue of muscle regenerative capacity. Importantly, our data uncovers that muscle stem cells in diabetic animals have reduced mitochondrial membrane potential, which is restored to its healthy levels by RSV that does not act via Sirt1, based on the pharmacological and genetic data but that this boost of intracellular energy enables productive tissue repair, but RSV in an animal model of type I diabetes.
\end{abstract}

Keywords: Resveratrol; Sirtuins; Muscle regeneration; Type I diabetes; Satellite cells; Stem cells; Mitochondrial membrane potential

Abbreviation: RSV: Resveratrol; BGL: Blood Glucose Level; IDDM:
Insulin-Dependent Diabetes; STZ: Streptozotocin; CTX: Cardiotoxin
I; DPI: Days Post Injury; INS: Insulin; TA: Tibialis Anterior; Gastroc: Gastrocnemius

\section{Introduction}

Millions of people suffer from diabetes mellitus, which manifests as a lack of cell and organ function, poor wound healing and reduced longevity due to dysfunction of many organ systems [1-5]. Type I or insulin-dependent diabetes (IDDM), is called juvenile because it mainly occurs in children, adolescents, or young adults; it is also an autoimmune disease [6]. Non-insulin-dependent (NIDDM), type II diabetes is typically caused by the insulin resistance or inadequate insulin signaling and is often linked to obesity [7]. Insulin controls glucose output by the liver and activates glucose transporters, enabling cells to uptake glucose from the blood to be metabolized in the Krebs cycle and/or glycolysis for ATP production in the mitochondria $[8,9]$. Consequentially, a lack of insulin or deficient insulin signaling deregulates energy metabolism, and one key biomarker as well as a hallmark of diabetes is elevated BGL [10].

Insulin has proven to be a life saving treatment in diabetic disorders because it restores glucose metabolism, and additional molecules that ameliorate the affects of perturbed glucose metabolism have been searched for $[11,12]$. Among such factors known to participate in the regulation of energy homeostasis in general and in cases of type II diabetes is RSV (3,4',5-trihydroxystilbene), a natural polyphenol with pleiotropic function. RSV influences several key molecules of the phosphatidyl inositol 3-kinase (PI3K)/Akt and mTOR signaling pathways (thus, RSV has anti-cancer properties), it also reduces oxidative damage to cells and acts in part by increasing the activity of
Sirt1. Sirtuins, including Sirt1, are NAD(+)-dependent deacetylases that control function of numerous proteins. Sirt1 increases fat metabolism and regulates mitochondrial function via the AMP kinase/Sirt1-PGC1a-PPAR network [13]. Both RSV and inducers of Sirt1 have shown recent promise as potential clinical targets for normalizing metabolism in type II diabetes [14,15]. Sirt1 plays a dual role in energy metabolism: it can both up- and down-regulate gluconeogenesis, which serves to maintain steady energy levels in the fasting state [14]. One of the targets of Sirt1 is PGC-1 $1 \alpha$, which positively regulates mitochondrial biogenesis and function. Interestingly, studies in mice with a muscle-specific Sirt1 knockout suggest that Sirt1 is not involved in RSV-induced mitochondrial biogenesis in muscle cells, indicating that other targets of RSV must be responsible for the positive effects of RSV on skeletal muscle metabolism [16].

While it is assumed that restoration of glucose metabolism is the key to curing diabetic disease, some of the manifestations of diabetes (e.g. deteriorated tissue maintenance and repair), can be ameliorated in the presence of high blood glucose levels, e.g. despite the ongoing

${ }^{*}$ Corresponding authors: Irina M. Conboy, 174 Stanley Hall, Department of Bioengineering, University of California Berkeley, Berkeley, CA, USA, Tel: (510) 666-2792; Fax: (510) 642-5835; E-mail: iconboy@berkeley.edu

Jaemin Jeong, Division of Radiation Caner Research, Korea Institute of Radiological \& Medical Science, 215-4 Gongneung-Dong, Nowon-Gu, Seoul, 139-706, South of Korea, Tel : +82-10-4712-5256; Fax: +82-2-970-2402; E-mail: jmj1103@gmail.com

Received July 18, 2013; Accepted September 11, 2013; Published September 17, 2013

Citation: Jeong J, Conboy MJ, Conboy IM (2013) Sirt1-Independent Rescue of Muscle Regeneration by Resveratrol in Type I Diabetes. J Diabetes Metab 4: 289. doi:10.4172/2155-6156.1000289

Copyright: ( 2013 Jeong J, et al. This is an open-access article distributed under the terms of the Creative Commons Attribution License, which permits unrestricted use, distribution, and reproduction in any medium, provided the original author and source are credited. 
hyperglycemia. Specifically, inhibition of myostatin/TGF- $\beta$ receptor/ pSmad3 rescues regeneration of skeletal muscle in a mouse model of type I diabetes [3]. When combined with insulin treatment, such additional ways to preserve tissue health might prove invaluable in the treatment of diabetes and other severe chronic metabolic disorders.

The direct influence of diabetes on the performance of tissue stem cells has just begun to be uncovered, and our recent work in type I diabetes demonstrated that a lack of insulin and resulting high blood glucose levels quickly but reversibly incapacitates the regenerative responses of muscle stem cells [3], which can to some extent explain the phenomenon of muscle wasting in cases of diabetes [17,18]. Muscle stem cells (also known as satellite cells) are typically quiescent and start dividing in response to injury or attrition of muscle fibers (myofibers); activated satellite cells then differentiate along the myogenic lineage into fusion-competent proliferating myoblasts (expressing Pax7, MyoD and desmin) and postmitotic multinucleated myotubes (expressing eMyHC), which repair the damaged tissue [19]. Based on our work, type I diabetes has a direct negative effect on the ability of muscle stem cells to activate and regenerate muscle, due to intensified myostatin/ TGF- $\beta$ receptor/pSmad 3 signaling [3]. Both exogenous insulin and inhibitors of the TGF- $\beta$ receptor rescue the regenerative capacity of muscle stem cells in the diabetic condition to $\sim 80 \%$ of healthy controls, and interestingly, as mentioned above, attenuation of TGF- $\beta$ receptor signaling enables robust muscle repair in the diabetic state [3].

To further understand the reasons behind poor activation of muscle stem cells in the diabetic organism and to uncover additional ways for improved tissue regeneration in cases of this metabolic disorder, we studied mitochondrial function in muscle stem cells resident to control mice versus animals in which the pancreatic $\beta$-islet cells were ablated by a single application of streptozotocin, STZ [20]. We hypothesized that the lack of intracellular glucose and resulting poor intracellular energy metabolism in this type I diabetes model might directly suppress the responses of organ stem cells (e.g., muscle stem cells), which require high levels of ATP in order to engage in such energy-consuming processes as proliferation and lineage-specific differentiation.

Indeed our data demonstrate that mitochondrial membrane potential is significantly diminished in satellite cells isolated from mice that model type I diabetes. Furthermore, our results establish that RSV, delivered systemically to mice lacking $\beta$-islet cells, rescues the regenerative capacity of satellite cells and restores productive muscle repair in the diabetic animals. And the positive effects of RSV are manifested through the enhancement of mitochondrial membrane potential, e.g. by restoring the intracellular energy homeostasis in satellite cells, even while blood glucose levels remain high.

\section{Materials and Methods}

\section{Mice}

Control (C57 BL/6, 2-4 month) male mice were obtained from Jackson Laboratories (Bar Harbor, ME, USA). Animals were housed and cared for under the UC Berkeley Office of Laboratory Animal Care.

\section{STZ, insulin, RSV and EX-527 administration}

Control mice were given a single intraperitoneal injection (IP) of STZ (Sigma-Aldrich, MO, USA) at a dose of $50 \mathrm{mg} / \mathrm{kg}$ body weights, or PBS injection for controls. After one weeks of STZ treatment, blood glucose level was measured using One Touch UltraMini (LifeScan, CA). Animals showing blood glucose level $>300 \mathrm{mg} / \mathrm{dl}$ were considered hyperglycemic. Hyperglycemic mice were given intraperitoneal injections of insulin (0.75 U/kg, Sigma-Aldrich, MO), RSV ( $3 \mathrm{mg} / \mathrm{kg}$, Sigma-Aldrich, MO) and/or EX527 (100 $\mu$ l of $100 \mu \mathrm{M}$ stock per mouse, Calbiochem/ EMD Millipore, CAS Number 49843-98-3) once a day for 3-5 days after the induction of hyperglycemia.

\section{Muscle injury}

Isoflurane was used to anesthetize the animals during the muscle injury procedure, as previously described [3]. Briefly, for bulk myofiber satellite cell activation, tibialis anterior and gastrocnemius muscles were injected with Cardiotoxin I (CTX, Sigma-Aldrich, MO) dissolved at $100 \mu \mathrm{g} / \mathrm{ml}$ in PBS, at 2-5 sites for each muscle, for a total of $50 \mu \mathrm{l} / \mathrm{g}$ muscles. Muscles were then harvested 3 days later. For lentiviral shRNA co-administration, $10 \mu \mathrm{l}$ cardiotoxin was diluted with $86 \mu \mathrm{l}$ of shRNA of Sirt1 lentivius $\left(1 \times 10^{6}\right.$ titer, Mission shRNA Lentiviral Transduction Particles Sigma-Aldrich, Mo) and $4 \mu \mathrm{g} / \mathrm{ml}$ Polyprene to give $100 \mu \mathrm{g} / \mathrm{ml}$ cardiotoxin and injected as above. For focal injury to assay regeneration in vivo, $2-3 \mu \mathrm{l}$ of $1 \mu \mathrm{g} / \mathrm{ml}$ CTX was injected at one site to the middle of the tibialis anterior or two sites to the gastrocnemius, and muscle harvested 5 DPI.

\section{Isolation of myofibers and satellite cells}

Myofibers and satellite cells were purified as described [21]. Briefly, dissected muscle were digested for $1 \mathrm{~h} 30 \mathrm{~min}$ at $37^{\circ} \mathrm{C}$ in DMEM with $1 \%$ penicillin-streptomycin, 250 units $/ \mathrm{ml}$ Collagenase Type IIA (Sigma-Aldrich, MO) and dissociated into myofibers with attached satellite cells by trituration, sedimentation and washing. Satellite cells were liberated from the fibers by digestion for 1 hour in DMEM with 40 units $/ \mathrm{ml}$ Collagenase type II and 2 units/ml Dispase, followed by sedimentation, filtration and washing. Leukocytes were depleted using anti-CD45-coated magnetic beads. This procedure yields 95\% pure satellite cells, based on their functional activity [22]. Sirt1 knockdown effects were confirmed by western blot using Sirt1 antibody (Santa Cruz Biothecnology, Inc., CA)

\section{Satellite cell myogenic potential}

Activated in vivo myofiber-associated satellite cells were cultured in growth medium (GM) containing Ham's F10 (Mediatech, VA), $20 \%$ Bovine growth serum (HyClone, IL), $5 \mathrm{ng} / \mathrm{ml}$ bFGF (Invitrogen, $\mathrm{CA}$ ) and 1\% Penicillin/streptomycin (Invitrogen, CA) on ECM-coated plates (1:500; BD Biosciences, CA) for 24 hours. GM was replaced daily. After addition of BrdU (10 $\mu \mathrm{M}$, Sigma-Aldrich, MO) to the GM for 2 hours, the cultures were switched into differentiation medium (DM, DMEM containing 2\% Horse serum and 1\% Penicillin/streptomycin) for 48 hours, in order to assay generation of de-novo myotubes, which are typically produced by the proliferative fusion-competent myoblasts in DM.

\section{Muscle repair assays by immunofluorescence and histological analysis}

All muscle tissue was dissected, flash frozen in OCT compound (Tissue Tek, Japan), cryo-sectioned at $10 \mu \mathrm{m}$, as previously described [3]. Muscle sections were stained with aqueous hematoxylin and eosin (H\&E; Sigma-Aldrich, MO) and immunostaining was performed as described [3]. Briefly, after permeabilization in PBS containing 1\% FBS and $0.25 \%$ Triton-X-100, tissues and cells were incubated with $1 \mu \mathrm{g}$ of primary antibodies per $\mathrm{ml}$ of staining buffer (PBS containing $1 \%$ FBS) for 1 hour at room temperature, followed by washes with staining buffer and then by 1 hour incubation with a $1 \mu \mathrm{g} / \mathrm{ml}$ dilution of secondary, fluorochrome labeled antibodies (ALEXA, Invitrogen, CA) in staining buffer. BrdU-specific immunostaining required an extra 
step of $2 \mathrm{M} \mathrm{HCl}$ treatment (denaturation of DNA) for 30 min before the permeabilization step.

\section{Measurement of mitochondrial membrane potential}

Isolated satellite cells were resuspended in $1 \mathrm{ml}$ DMEM medium without phenol red (Mediatech) containing $2 \mu \mathrm{M}$ JC-1 (5,5',6,6'-tetrachloro-1,1',3,3'-tetraethylbenzimidazolo-carbocyanine iodide, Molecular Probes). After incubation at $37^{\circ} \mathrm{C}$ for $10 \mathrm{~min}$, cells were washed with PBS and resuspended with DMEM without phenol red. Cells were analyzed by flow cytometry (FACScan, Beckman Coulter Epics XL), using $488 \mathrm{~nm}$ excitation with $525 \mathrm{~nm}$ for JC-1 green and $575 \mathrm{~nm}$ for JC-1 red band pass emission filters. Mitochondrial depolarization was indicated by a decrease in the red/green fluorescence intensity ratio [23].

\section{Results}

\section{Systemic RSV rescues muscle regeneration in type I diabetes}

Type I diabetes was modeled in young wild type mice by ablation of $\beta$-islet cells with STZ; untreated mice of the same genotype and matched by sex and age were used as healthy controls. After a single injection of $180 \mathrm{mg} / \mathrm{kg}$ of STZ, blood glucose levels were monitored daily and at the first occurrence of high BGLs, skeletal muscle (Tibialis Anterior, TA and Gastrocneimus, Gastroc) of STZ-treated mice were injured by cardiotoxin, CTX (as described in Methods and previously published) [3]. Healthy control mice were monitored for BGLs and were injured by CTX identically and in parallel with the STZ-treated cohort. Prior to the administration of STZ and without STZ treatment the BGLs were in the 110 to $130 \mathrm{mg} / \mathrm{dl}$ range, and following the ablation of $\beta$-islet cells by STZ, the BGLs were over $300 \mathrm{mg} / \mathrm{dl}$.

To test the effects of RSV on muscle regeneration, some of the mice administered with STZ were also treated with systemic application of RSV. Specifically, RSV was injected intraperitoneally (IP) into STZtreated mice everyday (STZ/RSV) until muscle harvesting. The BGLs of STZ/RSV mice were still over $300 \mathrm{mg} / \mathrm{dl}$.

Two well-published assays of myogenic capacity were used to evaluate the regenerative performance of muscle stem cells [19]. In the first assay, myofiber-associated satellite cells activated by muscle injury were isolated 3 days post injury (DPI) and assayed ex-vivo for their proliferation, followed by differentiation into de-novo myotubes. In this assay the properties of satellite cells that are established in vivo are monitored soon after the isolation of these cells from skeletal muscle tissue. Specifically, myofiber explant cultures were kept in growth medium for 24 hours, BrdU was added for the last two hours to label proliferating cells, after which cultures were switched to mitogen-low differentiation medium for 48 hours and the numbers of $\mathrm{eMyHC}^{+}$ multinucleated myotubes with $\mathrm{BrdU}^{+}$central nuclei (e.g. differentiated progeny of activated satellite cells) were quantified. As compared to healthy controls, the diabetic condition (STZ) resulted in the severely diminished myogenic capacity of muscle stem cells; however, systemic RSV significantly restored the regenerative responses of satellite cells even in mice with ablated $\beta$-islet cells (Figure 1A, quantified in 1B).

In the second assay, TA and Gastroc muscle was isolated at 5 DPI, from Control, STZ and STZ/RSV mice. Tissue was cryosectioned and the numbers of de-novo eMyHC ${ }^{+}$myofibers with centrally located nuclei were determined by $\mathrm{H} \& \mathrm{E}$ histology and immunofluorescence and were quantified throughout the injury sites. As shown in Figures $1 \mathrm{C}$ and $1 \mathrm{D}$, muscle repair in vivo declined in STZ mice but was robustly and significantly improved by the systemic administration of RSV.
These data suggest that in agreement with our previous work, the diabetic condition quickly and severely incapacitates muscle regeneration, and demonstrate that systemically administered RSV is effective in restoring regenerative responses to muscle stem cells and improving the repair of muscle damage in the presence of diabetes and hyperglycemia.

\section{RSV enhances muscle regeneration in type I diabetes in sirt1- independent fashion}

According to previous reports, a Sirtuin protein, Sirt1, enhances proliferation of cultured muscle precursor cells by decreasing the cell cycle inhibitor p21 [24], and by slowing down the rate of muscle

A

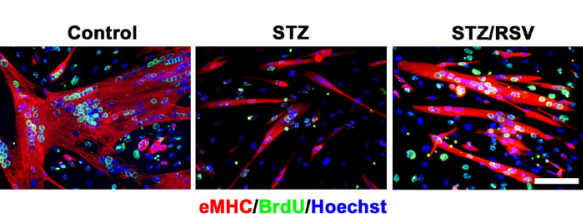

B

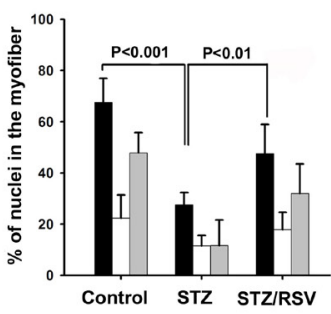

C

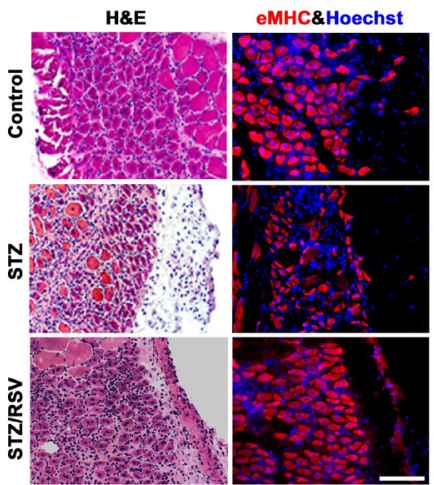

D

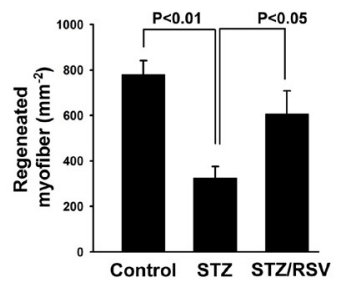

Figure 1: Satellite cell myogenic potential and muscle regeneration by RSV in STZ-treated control mice. (A) Satellite cells were prepared from tibialis anterior (TA) and gastrocnemius (Gastroc) muscle of control, STZ-treated mice (STZ), and RSV-treated STZ mice (STZ/RSV) at 3 days post-injury (DPI), cultured in growth medium for $24 \mathrm{~h}$ and then switched to differentiation medium for $48 \mathrm{~h}$. Formation of de-novo eMyHC+ myotubes (red) and $\mathrm{BrdU}^{+}$nuclei (green) were assayed by immunofluorescence. Hoechst (blue) labels all nuclei. Scale bar, 100 $\mu \mathrm{m}$. (B) The percent of nuclei in $\mathrm{eMyHC}^{+}$myotubes to total nuclei (black bars), the percent of $\mathrm{BrdU}^{+}$nuclei in myotubesto total nuclei (white bars), and the percent of $\mathrm{eMyHC}^{+}$myotubes with more than two nuclei to total nuclei (gray bars)were quantified. $n=3$ with more than three independent experiments; $P<0.001$ control as compared to STZ-treated mice, and $P<0.01$ STZ/RSV as compared to STZtreated mice. Data are mean $\pm S D$. (C) Assessment of muscle regeneration in vivo was performed by hematoxylin and eosin (H\&E) histological analysis and by immunodetection of de-novo eMyHC+ myofibers (Red) in $10 \mu \mathrm{m}$ cryosections of TA and Gastroc muscles that were isolated at $5 \mathrm{DPI}$. Scale bar, $100 \mu \mathrm{m}$. (D) Numbers of de-novo myofibers per $\mathrm{mm}^{2}$ of injured muscle (covering the entire injury site) were quantified. $n=3$ with more than three independent experiments; $\mathrm{P}<0.01$ control as compared to STZ-treated mice, and $\mathrm{P}<0.05 \mathrm{STZ} / \mathrm{RSV}$ as compared to STZ treated mice. Data are mean \pm SD. 
differentiation [25]. Additionally, RSV is a known inducer of Sirt1 and both molecules have been shown to improve energy homeostasis in cases of type II diabetes $[13,14]$. Based on these data, we decided to determine whether the positive effects of RSV and of ectopic insulin on satellite cell regenerative responses are mediated by Sirtl and hence, would be diminished by Sirt1 lack of function. We used two independent approaches for the inhibition of Sirt1: genetic downmodulation by shRNA (delivered in vitro to freshly isolated satellite cells), and pharmacological attenuation by a small molecule EX-527 delivered in vivo to mice. As shown in Figure 2A, Sirt1 levels were effectively down regulated in muscle stem cells by lentivirally delivered shRNA against Sirt1. Interestingly, down-regulation of Sirtl by the shRNA in satellite cells derived from either healthy controls or STZ/ INS or STZ/RSV-treated mice did not cause a significant reduction of myogenic proliferation and differentiation (Figures $2 \mathrm{~B}$ and $2 \mathrm{C}$ ). In agreement with previous publications and data shown above, the robust myogenic capacity of satellite cell cultures (determined based on the ability to form de-novo myotubes), was found to decline in cells derived from STZ-treated mice and was restored to healthy levels by the in vivo application of insulin or RSV (Figures 2B-2E). Notably, these robust regenerative responses of the satellite cells were independent of Sirt1 (in all tested conditions), as evident from Figures 2B-2E. Moreover, a pharmacological in vivo approach confirmed these conclusions: administration of specific pharmacological inhibitor of Sirt1, EX-527, to live animals did not affect the regenerative capacity of muscle stem cells derived from diabetic STZ-treated mice (Figures 2D and 2E). Similarly to the shRNA targeting, this in vivo pharmacological inhibition of Sirt1 did not interfere with the myogenesis of satellite cells that were derived from all studied cohorts: Control, STZ, STZ/INS and STZ/RSV treated animals (Figures 2D and 2E).

These results suggest that the regenerative potential of muscle stem cells that are resident to animals which model type I diabetes are restored by RSV and Insulin in a Sirt1 independent manner. Moreover, inhibition of Sirtl did not negatively impact the performance of satellite cells derived from healthy untreated controls. Therefore, Sirt1 does not appear to be involved in physiologic muscle regeneration and the rescue of myogenicity in this mouse model of type I diabetes occurs without significant involvement of Sirtl.

\section{Mitochondrial membrane potential is diminished in muscle stem cells from hyperglycemic mice, and becomes restored by RSV}

RSV is known to improve energy homeostasis [16] and so we hypothesized that the mechanism of the positive effects of RSV on the myogenicity of satellite cells is linked to mitochondrial function. This hypothesis also implies that a decline in mitochondrial activity is one of the key causes of poor regenerative performance of muscle stem cells in a diabetic environment. To explore these hypotheses, we analyzed the mitochondrial membrane potential in satellite cells that were freshly isolated from the healthy control, STZ treated and STZ/RSV treated mice.

Muscle stem cells were isolated from TA and Gastroc muscle at 3 days after CTX-induced injury and assayed for their mitochondrial membrane potential right after derivation. To measure the mitochondrial membrane potential, we used the JC-1 assay kit, in which JC1 label is detected as green (present in the cytosol) versus red fluorescence (accumulates in the mitochondria) [23,26].

Very interestingly, while satellite cells were viable in all the studied conditions, cells derived from mice that had STZ-induced ablation of
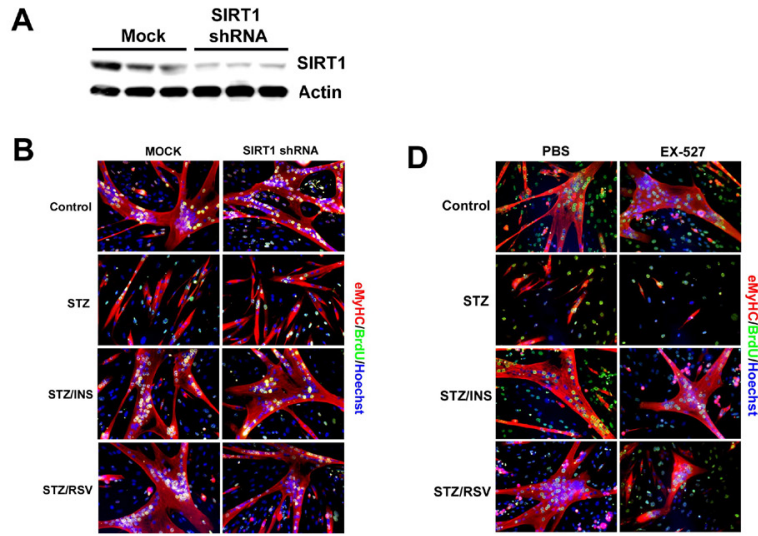

C

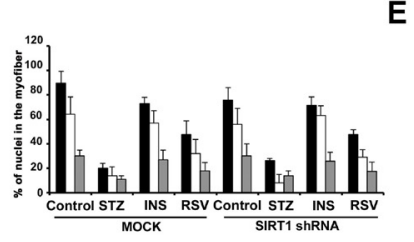

E

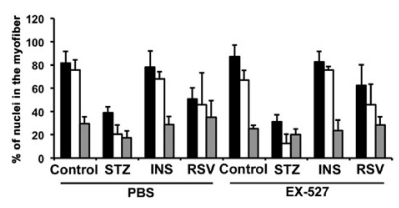

Figure 2: Muscle regeneration in type I diabetes by RSV in a Sirt1-independent manner. (A) Western blotting of myoblasts transfected with lentiviral particles delivering Sirt1 shRNA shows effective knock down of Sirt1 protein expression; $n=3$ separate cultures. (B) Satellite fiber-associated cells were prepared from TA and Gastroc muscle of control, STZ-treated mice (STZ), insulin-treated STZ mice (STZ/INS) and RSV-treated STZ mice (STZ/RSV) at 3 DPI with or withoutSirt1 shRNA infection, cultured in growth medium for $24 \mathrm{~h}$, pulsed with BrdU for 2 hours and then switched to differentiation medium for $48 \mathrm{~h}$. Formation of de-novo eMyHC+myotubes (Red) and $\mathrm{BrdU}^{+}$nuclei (Green) were assayed by immunofluorescence. Hoechst (Blue) labels all nuclei. Scale bar, $100 \mu \mathrm{m}$. (C) The percent of nuclei in eMyHC $\mathrm{HC}^{+}$myotubes to total nuclei (black bars), the percent of $\mathrm{eMyHC}^{+}$myotubes with more than two nuclei to total nuclei (white bars), and the percent of $\mathrm{BrdU}^{+}$nuclei in myotubes to total nuclei (gray bars) were quantified. $n=3$ with more than three independent experiments. (D) Satellite cells were prepared from TA and Gastroc muscle of control, STZ-treated mice (STZ), inuslin-treated STZ mice (STZ/INS) and RSV-treated STZ mice (STZ/RSV) at $3 \mathrm{DPI}$ with EX-527 administration, cultured in growth medium for $24 \mathrm{~h}$ and then switched to differentiation medium for $48 \mathrm{~h}$. Formation of de-novo eMyHC+ myotubes (Red) and BrdU+ nuclei (Green) were assayed by immunofluorescence. Hoechst (Blue) labels all nuclei. Scale bar, $100 \mu \mathrm{m}$. (E) The percent of nuclei in $\mathrm{eMyHC}^{+}$myotubes to total nuclei (black bars), the percent of eMyHC+myotubes with more than two nuclei to total nuclei (white bars), and the percent of $\mathrm{BrdU}^{+}$ nuclei in myotubes to total nuclei (gray bars) were quantified. $n=3$ with more than three independent experiments.

pancreatic $\beta$-cells had a significant decline in mitochondrial membrane potential, as compared to the cells isolated from the healthy, untreated control mice (Figure 3A, quantified in 3B). Notably, the administration of exogenous RSV systemically in vivo, rescued the mitochondrial function in muscle stem cells that were derived from STZ-treated mice, almost to the levels of healthy controls (Figures 3A and 3B).

These results demonstrate that mitochondrial membrane potential declines in muscle stem cells residing in a diabetic environment, and suggest that restoring energy homeostasis by RSV administered in vivo, improves mitochondrial function and the regenerative capacity of these organ stem cells.

\section{Discussion}

This work is the first to demonstrate the direct and immediate effects of diabetic insulin loss and hyperglycemia on the mitochondrial activity of muscle stem cells. It is quite possible that responses of 

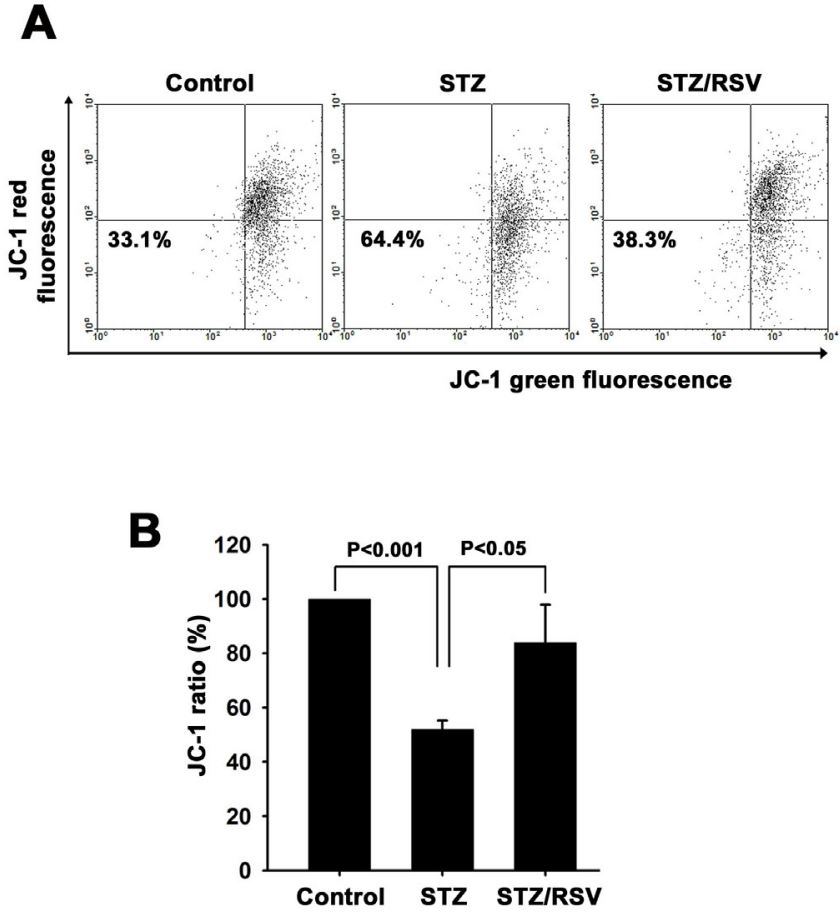

Figure 3: Mitochondrial membrane potential restored by RSV administration. (A) Mitochondrial membrane potentials of TA and Gastroc muscle satellite cells derived from control, STZ-treated, and STZ/RSV-treated mice at 3DPI, were measured by FACS analysis using the JC-1 assay kit. (B) The percent of $\mathrm{JC}-1 \mathrm{red} / \mathrm{green}$ ratio was quantified, $n=3$ with more than three independent experiments; $\mathrm{P}<0.001$ control as compared to STZ-treated mice, and $\mathrm{P}<0.05$ STZ/RSV as compared to STZ-treated mice. Data are mean \pm SD.

tissue stem cells in general and not just in skeletal muscle become incapacitated in diabetic environments, due to the lack of intracellular glucose resulting in diminished mitochondrial function. In this regard, it was shown that under ischemia, neural stem cells die from glucose deprivation rather than from oxygen deprivation [27]. The finding that organ stem cells are likely to be acutely sensitive to glucose deprivation implies that the multiple tissue degenerative disorders that accompany diabetes are likely to be in large part caused by the inhibition of their stem cell responses.

The lack of the regenerative responses of the muscle stem cells that were isolated from diabetic animals is persistent and manifests even after 72 hours of culture in the presence of insulin in the culture medium (Figures 1 and 2); these data imply that even a brief diabetic state might cause a lasting defect in tissue maintenance and repair. Consequentially, these results emphasize the need for a combinatorial therapy to improve tissue regeneration in the diabetic condition, where some of the treatments are dedicated to enhance tissue repair in the presence of, and recovering from, hypoinsulemia and hyperglycemia.

The rescue of muscle regeneration by RSV in mice with ablated $\beta$-islet cells and manifesting high BGLs (Figure 1) is the first demonstration of positive effects of RSV on stem cells in type I diabetes. Such an improvement in the ability of satellite cells resident to the diabetic organism to repair muscle damage was further confirmed by the enhanced myogenic capacity of these muscle stem cells ex vivo (Figures 1 and 2). Very interestingly, Sirt1,the logical candidate for the RSV effects on muscle stem cells, was not found to be significantly involved, and thus other down-stream effectors of RSV will need to be studied in order to more completely understand the mechanism by which RSV enhances myogenesis in an animal model of type I diabetes. In this regard, RSV was delivered systemically to live animals and thus its positive effects on muscle stem cells could be indirect, e.g. via the global improvement in energy metabolism and reduced protein degradation, which have been reported in a rat STZ model [28]. Regardless of a precise molecular mechanism, from a clinical perspective this work uncovers a novel strategy for improving the regenerative performance of organ stem cells in type I diabetes.

In search of the molecular mechanisms responsible for the decline in muscle regeneration, we have identified reduced mitochondrial membrane potential in the satellite cells of the diabetic animals as a critical factor (Figure 3). Very interestingly, in vivo administered RSV resulted in the enhancement of the mitochondrial membrane potential in satellite cells derived from STZ-treated mice, in which $\beta$-islet cells were ablated and BGLs were very high (Figure 3 ), and this is consistent with the rescue of myogenic responses by RSV (Figures 1 and 2). A transient (days to a week) down-modulation of Sirt1 did not affect the regenerative capacity of muscle stem cells, and the rescue of muscle regeneration by RSV seems to be independent of Sirt1 in this experimental system (Figure 2). However, a prolonged change in glucose metabolism might act through the RSV/Sirt1 axis, and similarly, long-term inhibition of Sirt1 activity might negate the positive effects of RSV on mitochondrial function and on the regenerative responses of tissue stem cells, both of which would be very interesting to study in the future.

Summarily, our data are most consistent with the conclusion that within days of reduced intracellular glucose, mitochondrial function rapidly declines in muscle stem cells in the diabetic environment, causing diminished proliferation and differentiation, and restoring mitochondrial activity in these satellite cells by in vivo administered RSV enhances muscle regeneration. The poor muscle stem cell responses that are caused by the decline in mitochondrial activity are further exacerbated by the elevated levels of the physiologic inhibitor myostatin in the stem cell niche, which results in intensified TGF- $\beta$ receptor/Smad3 signaling [3]. As such, several independent, inhibitory mechanisms prevent the activation and proliferation of muscle stem cell in the diabetic environment.

Another interesting implication of the current study is that the rapid and direct influence of deregulated glucose homeostasis observed as a decline in cell cycle and performance of organ stem cells, suggest that the longevity (life span) of a mammal that relies on continuous tissue regeneration may require well-regulated insulin signaling. Inactivating mutations in the insulin/IGF-1 endocrine pathway reduce the rate of metabolism, induce expression of stress response genes and extend life-span, particularly in C. elegans and Drosophila $[29,30]$. But note these long-lived mutants lacking insulin/IGF-1 activity are in species where the adult animals are composed almost entirely of post-mitotic cells. In mice, where there are separate and promiscuous insulin and IGF-1 signaling pathways, a partial decrease in IGF-1 or growth hormone, a lack of insulin receptors in adipose tissue, or heterozygosity or lack of IRS-2 (in general or in brain) have a much less robust and at times a gender-specific positive effect on life span [31-33]. The organism-wide glucose metabolism remains unperturbed in these experimental models of reduced insulin/IGF-1 signaling [29]. Therefore, while differentiated cells might benefit from decreased insulin signaling through lowered metabolic rate and enhanced stressresponses [34], the high energy requirements of organ stem cells during tissue turn-over or regeneration make them more dependent on robust insulin signaling and balanced glucose uptake. Thus tissue attrition and 
Citation: Jeong J, Conboy MJ, Conboy IM (2013) Sirt1-Independent Rescue of Muscle Regeneration by Resveratrol in Type I Diabetes. J Diabetes Metab 4: 289. doi:10.4172/2155-6156.1000289

degeneration would be inevitable in a mammal that does not maintain glucose metabolism precisely, and this would likely limit the extent of reducing insulin signaling as an evolutionary adaptation to delay aging.

\section{References}

1. Furie K, Inzucchi SE (2008) Diabetes mellitus, insulin resistance, hyperglycemia, and stroke. Curr Neurol Neurosci Rep 8: 12-19.

2. Botusan IR, Sunkari VG, Savu O, Catrina AI, Grünler J, et al. (2008) Stabilization of HIF-1alpha is critical to improve wound healing in diabetic mice. Proc Nat Acad Sci U S A 105: 19426-19431.

3. Jeong J, Conboy MJ, Conboy IM (2013) Pharmacological inhibition of myostatin/ TGF- $\beta$ receptor/pSmad3 signaling rescues muscle regenerative responses in mouse model of type 1 diabetes. Acta Pharmacol Sin 34: 1052-1060.

4. Banks AS, Kon N, Knight C, Matsumoto M, Gutiérrez-Juárez R, et al. (2008) SirT1 gain of function increases energy efficiency and prevents diabetes in mice. Cell Metab 8: 333-341.

5. Zimmet $P$, Alberti KG, Shaw J (2001) Global and societal implications of the diabetes epidemic. Nature 414: 782-787.

6. Cooke DW, Plotnick L (2008) Type 1 diabetes mellitus in pediatrics. Pediatr Rev 29: 374-384

7. Vijan S (2010) Type 2 diabetes. Ann Intern Med 152: ITC31-15

8. MacDonald PE, Joseph JW, Rorsman P (2005) Glucose-sensing mechanisms in pancreatic beta-cells. Philos Trans R Soc Lond B Biol Sci 360: 2211-2225.

9. Lloyd D, Lemar KM, Salgado LE, Gould TM, Murray DB (2003) Respiratory oscillations in yeast: mitochondrial reactive oxygen species, apoptosis and time; a hypothesis. FEMS Yeast Res 3: 333-339.

10. Sonksen $P$, Sonksen J (2000) Insulin: understanding its action in health and disease. Br J Anaesth 85: 69-79.

11. Lestradet $H$ (1997) [The 75th anniversary of the discovery of insulin]. Diabetes Metab 23: 112-117.

12. Ravaine V, Ancla C, Catargi B (2008) Chemically controlled closed-loop insulin delivery. J Control Release 132: 2-11.

13. Morris BJ (2013) Seven sirtuins for seven deadly diseases of aging. Free Radic Biol Med 56: 133-171.

14. Kitada M, Kume S, Kanasaki K, Takeda-Watanabe A, Koya D (2013) Sirtuins as possible drug targets in type 2 diabetes. Curr Drug Targets 14: 622-636.

15. Balestrieri ML, Servillo L, Esposito A, D'Onofrio N, Giovane A, et al. (2013) Poor glycaemic control in type 2 diabetes patients reduces endothelial progenitor cell number by influencing SIRT1 signalling via platelet-activating factor receptor activation. Diabetologia 56: 162-172.

16. Menzies KJ, Singh K, Saleem A, Hood DA (2013) Sirtuin 1-mediated effects of exercise and resveratrol on mitochondrial biogenesis. J Biol Chem 288: 69686979.

17. Vignaud A, Ramond F, Hourdé C, Keller A, Butler-Browne G, et al. (2007) Diabetes provides an unfavorable environment for muscle mass and function after muscle injury in mice. Pathobiology 74: 291-300.
18. Krause MP, Riddell MC, Hawke TJ (2011) Effects of type 1 diabetes mellitus on skeletal muscle: clinical observations and physiological mechanisms. Pediatr Diabetes 12: 345-364.

19. Conboy IM, Rando TA (2012) Heterochronic parabiosis for the study of the effects of aging on stem cells and their niches. Cell Cycle 11: 2260-2267.

20. Förster O, Rudas B (1969) Ketosis in rats with streptozotocin-induced diabetes Lancet 1: 1321-1322.

21. Conboy MJ, Conboy IM (2010) Preparation of adult muscle fiber-associated stem/precursor cells. Methods Mol Biol 621: 149-163.

22. Sherwood ER, Enoh VT, Murphey ED, Lin CY (2004) Mice depleted of CD8+ T and NK cells are resistant to injury caused by cecal ligation and puncture. Lab Invest 84: 1655-1665.

23. Smiley ST, Reers M, Mottola-Hartshorn C, Lin M, Chen A, et al. (1991) Intracellular heterogeneity in mitochondrial membrane potentials revealed by a J-aggregate-forming lipophilic cation JC-1. Proc Natl Acad Sci U S A 88 : 3671-3675.

24. Rathbone CR, Booth FW, Lees SJ (2009) Sirt1 increases skeletal muscle precursor cell proliferation. Eur J Cell Biol 88: 35-44.

25. Fulco M, Schiltz RL, lezzi S, King MT, Zhao P, et al. (2003) Sir2 regulates skeletal muscle differentiation as a potential sensor of the redox state. Mol Cell 12: $51-62$

26. Cossarizza A, Baccarani-Contri M, Kalashnikova G, Franceschi C (1993) A new method for the cytofluorimetric analysis of mitochondrial membrane potential using the J-aggregate forming lipophilic cation $5,5^{\prime}, 6,6^{\prime}$-tetrachloro1,1',3,3'-tetraethylbenzimidazolcarbocyanine iodide (JC-1). BiochemBiophys Res Commun 197: 40-45.

27. Horie N, Moriya T, Mitome M, Kitagawa N, Nagata I, et al. (2004) Lowered glucose suppressed the proliferation and increased the differentiation of murine neural stem cells in vitro. FEBS Lett 571: 237-242.

28. Chen KH, Cheng ML, Jing YH, Chiu DT, Shiao MS, et al. (2011) Resveratro ameliorates metabolic disorders and muscle wasting in streptozotocin-induced diabetic rats. Am J Physiol Endocrinol Metab 301: E853-863.

29. Kenyon C (2005) The plasticity of aging: insights from long-lived mutants. Cell 120: $449-460$.

30. Laron $Z$ (2008) The GH-IGF1 axis and longevity. The paradigm of IGF1 deficiency. Hormones (Athens) 7: 24-27.

31. Hayashi T, Boyko EJ, McNeely MJ, Leonetti DL, Kahn SE, et al. (2008) Viscera adiposity, not abdominal subcutaneous fat area, is associated with an increase in future insulin resistance in Japanese Americans. Diabetes 57: 1269-1275.

32. Valverde AM, Fabregat I, Burks DJ, White MF, Benito M (2004) IRS-2 mediates the antiapoptotic effect of insulin in neonatal hepatocytes. Hepatology 40 : 1285-1294.

33. Taguchi A, Wartschow LM, White MF (2007) Brain IRS2 signaling coordinates life span and nutrient homeostasis. Science 317: 369-372.

34. Maiese K (2008) Diabetic stress: new triumphs and challenges to maintain vascular longevity. Expert Rev Cardiovasc Ther 6: 281-284

This article was originally published in a special issue, Type 1 Diabetes Mellitus handled by Editor. Dr. Larry Distiller, Centre for Diabetes \& Endocrinology (Pty) Ltd, South Africa 